\title{
Setting Research Priorities Within Allied Health: What Do Clinicians Think?
}

Annette Davis

Monash Health and Monash University, annette.davis@monash.edu

Den-Ching Angel Lee

Monash University, Australia, angel.lee@monash.edu

Lisa-Anabell Wenzel

Hochschule Hannover - University of Applied Sciences and Arts, lisa.wenzel@gmx.de

Terry Haines

Monash University, Terrence.Haines@monash.edu

Follow this and additional works at: https://nsuworks.nova.edu/ijahsp

Part of the Health and Medical Administration Commons

\section{Recommended Citation}

Davis A, Lee D, Wenzel L, Haines T. Setting Research Priorities Within Allied Health: What Do Clinicians Think?. The Internet Journal of Allied Health Sciences and Practice. 2019 Jan 01;17(1), Article 6.

This Manuscript is brought to you for free and open access by the College of Health Care Sciences at NSUWorks. It has been accepted for inclusion in Internet Journal of Allied Health Sciences and Practice by an authorized editor of NSUWorks. For more information, please contact nsuworks@nova.edu. 


\title{
Setting Research Priorities Within Allied Health: What Do Clinicians Think?
}

\begin{abstract}
Background: Research activity among allied health clinicians working in publicly funded health care organisations is growing, yet little attention has been paid to how these activities are prioritised. Without a specific framework to guide research direction, it is likely that research will be conducted in areas that are prioritised by individual clinicians. However, it is presently unknown what areas are prioritised by clinicians and the reasoning behind their prioritisation. Purpose: The aims of this study were to identify what clinicians identify to be the highest priority research across personal, department, and health service levels, and the reasons why. It also sought to identify barriers and enablers to conducting this research. Methods: Qualitative, cross-sectional survey with $n=95$ allied health clinician respondents within one organisation. Survey items relating to research priority were structured across three levels. Survey format consisted of open-ended questions and thematic content analysis was used to categorise responses. Results: The highest priority research across personal, departmental and organisational levels were related to areas of personal work, departmental service and models of care evaluation respectively. The top three priorities in all levels combined were "testing solutions", "understanding problems or developing solutions" and "implementing evidence-based approaches in real life". The primary reason provided as to why the research was considered to be of the highest priority was related to the anticipated effectiveness of intervention on patient outcomes and the efficiency of service delivery models being investigated. Time shortages/workload/competing priorities were the most frequently identified barriers to conducting this research, while mentoring /access to university partners/organisational culture/leadership and priorities that favoured research were the most frequently identified enablers. Conclusions: Considerations can be taken from our findings to guide the development of a framework or system to prioritise research projects in the publicly funded health care organisation.
\end{abstract}

\section{Author Bio(s)}

Annette Davis, (B.App.Sc(Pod)), is an Allied Health manager in Podiatry of Monash Health in Melbourne, Australia and a PhD candidate in the School of Primary and Allied Health Care of Faculty of Medicine, Nursing, and Health Sciences in Monash University, Melbourne, Australia. She is also a registered Podiatrist in Australia.

Den-Ching A. Lee, $(\mathrm{PhD})$, is the corresponding author. She is a Research Fellow in the School of Primary and Allied Health Care of Faculty of Medicine, Nursing, and Health Sciences in Monash University, Melbourne, Australia. She is also a registered Physiotherapist in Australia.

Lisa-Anabell Wenzel, (B.Sc.) is a Master degree student in Physiotherapy in the Faculty of Diaconic Studies, Health Care and Social Work of Hochschule Hannover - University of Applied Sciences and Arts, Hannover, Germany. She is also a practising Physiotherapist in Germany.

Terry P. Haines, (PhD), is the Head of School of Primary and Allied Health Care, Faculty of Medicine, Nursing, and Health Sciences of Monash University, Melbourne, Australia. He is a registered physiotherapist in Australia. 


\title{
IIJAHSP \\ The Internet Joưnal of Allied Health Sciences and Practice
}

Dedicated to allied health professional practice and education

Vol. 17 No. 1 ISSN 1540-580X

\section{Setting Research Priorities Within Allied Health: What Do Clinicians Think?}

\author{
Annette Davis ${ }^{1}$ \\ Den-Ching Angel Lee ${ }^{2}$ \\ Lisa-Anabell Wenzel ${ }^{3}$ \\ Terry Haines ${ }^{2}$
}

1. Monash Health and Monash University

2. Monash University

3. Hochschule Hannover

Australia and Germany

\begin{abstract}
Background: Research activity among allied health clinicians working in publicly funded health care organisations is growing, yet little attention has been paid to how these activities are prioritised. Without a specific framework to guide research direction, it is likely that research will be conducted in areas that are prioritised by individual clinicians. However, it is presently unknown what areas are prioritised by clinicians and the reasoning behind their prioritisation. Purpose: The aims of this study were to explore what clinicians identify to be the highest priority research across personal, department, and health service levels, and the reasons. It also sought to identify barriers and enablers to conducting this research. Methods: Qualitative, cross-sectional survey with n=95 allied health clinician respondents within one organisation. Survey items relating to research priority were structured across three levels. The survey format consisted of open-ended questions, and thematic content analysis was used to categorise responses. Results: The highest priority research across personal, departmental, and organisational levels were related to areas of personal work, departmental service, and models of care evaluation respectively. The top three priorities in all levels combined were "testing solutions," "understanding problems or developing solutions," and "implementing evidence-based approaches in real life." The primary reason provided as to why the research was considered to be of the highest priority was related to the anticipated effectiveness of intervention on patient outcomes and the efficiency of service delivery models being investigated. Time shortages/workload/competing priorities were the most frequently identified barriers to conducting this research, while mentoring laccess to university partners/organisational culture/leadership and priorities that favoured research were the most frequently identified enablers. Conclusions: Considerations can be taken from our findings to guide the development of a framework or system to prioritise research projects in the publicly funded health care organisation.
\end{abstract}

Keywords: priority, research, allied health, clinicians, reason, effectiveness 


\section{BACKGROUND}

Research activity by allied health clinicians working in public health services is growing. Recent government policy initiatives in Australia and the United Kingdom have enabled research capacity building in allied health through a coordinated, whole system approach with emphasis on research conducted through allied health, with allied health and by allied health. ${ }^{1-4}$ There has also been rapid growth in nationally competitive research funding grants being awarded to allied health professional groups. ${ }^{5}$

Not all research, however, is conducted through large, nationally competitive grant funding schemes. Publicly funded health services support research by allied health clinicians in a range of ways, such as employing allied health clinicians in research specific roles, supporting internal grant funding schemes, and making research a key performance indicator in some clinical position descriptions. ${ }^{6}$ Consequently, there is a growing amount of public funds being consumed through research conducted by allied health clinicians.

This raises the question as to whether these public resources are being directed towards projects that could be considered a high priority. There are different ways that a health service sector may identify research priority areas. For example, a sector may use a top-down approach or management-initiated research, and a bottom-up approach or clinician-initiated research. Both approaches may have advantages and disadvantages such as costs involved and relevance to clinicians' expertise, interests, and research skills. Currently, there are no formal systems or frameworks in place known to the authors to guide allied health clinicians, departments, and managers within publicly funded healthcare organisations in prioritising research to be conducted through their organisation. This is important, however, as there are limited resources available to support research within these organisations and a multitude of different ways in which these resources could be allocated. Without a framework of this nature, it is likely that research being pursued will follow the priorities of individual clinicians who initiate the research. Presently, it is unknown as to the types of projects that clinicians think should be prioritised, nor the reasons or principles underpinning why a particular project might be considered a high priority.

The aims of this study were to explore why allied health clinicians identify specific research projects as being a high priority for them personally, for their department or work area, and for their organisation health service sector. It also sought to categorise the different types of projects identified as being a high priority and to identify barriers and enablers to conducting these research projects.

\section{METHODS \\ Design}

This research was a qualitative investigation which took an exploratory approach. Data were collected through a cross-sectional survey which consisted of mostly open-ended questions. These questions asked respondents to identify the highest priority research projects they could think of and provide reasons for why they thought these projects would need to be undertaken at a personal level, across the department or work area, and the broader health service sector. Data for perceived barriers and enablers to the conduct of these research projects were also collected.

\section{Participants/setting}

Respondents of this survey were allied health clinicians from Monash Health. Monash Health is a public health service provider in Victoria, Australia. It provides hospital-based and community-based healthcare in the southeastern suburbs of Melbourne. ${ }^{7}$ The target group of the survey consisted of 750 allied health clinicians working across Monash Health.

\section{Survey Instrument}

The design of survey items was based on the research capacity and culture tool. ${ }^{8}$ It guided the structuring of the questionnaire into the three stages of research priority: personal, departmental, and organisational level.

Participants were asked to provide their responses in the PICO format ("patient," "intervention," "comparison," and "outcome") when they were asked what they thought would be the highest priority research. ${ }^{9}$ Examples of open-ended questions used were "Describe the highest priority project that could be undertaken by yourself (individually) if you were going to lead a research project. Remember to try and use the PICO format" and "Describe why you think this particular example of a project that could be undertaken by yourself (individually) is the highest priority for you." The same questioning was used to identify the highest priority research project that could be conducted at a "departmental" level and at the health care "organisational" level, and the reasons behind why they considered these projects to be a high priority. Participants were also asked, "What are the barriers that you perceive as stopping high priority research from being conducted at Monash Health?" and "What are the enablers that you perceive as facilitating high priority research to be conducted at Monash Health?" to capture data on the relevant barriers and enablers to conducting this research. A list of open-ended questions used in the survey is presented in Appendix 1. Demographic data were collected which included professional background and the highest education attained by each respondent. 


\section{Procedure}

The survey was approved by Monash Health Human Research Ethics committee (LNR/17/MonH/325). Informed consent to participate was implied through completion of the survey. The recruitment process and the completion of the survey occurred between July and August 2017.

Questions on the survey were loaded onto an online survey programme (www.surveymonkey.com, San Mateo, California, USA). An email including the link to the survey was sent to the allied health heads of professions at Monash Health in July 2017. They were asked to disseminate the questionnaire to allied health clinicians working in their department. Additionally, managers, team leaders, and senior staff were encouraged to discuss the importance of completing the survey with staff members. Respondents entered the responses directly onto the online survey.

\section{Data Analysis}

A thematic content analysis was undertaken. ${ }^{10}$ It was used to analyse the qualitative responses for what the respondents thought would be the highest priority research on a personal level and why, across their department or work area and the health service organisation. This same approach was applied to analyse the collected responses relating to what barriers and enablers respondents perceived to the conduct of these research projects.

This analytical approach first involved pooling of data and identifying specific areas of content in each area of interest. This content was then interpreted for its meaning to inform the categorisation and coding of responses. Themes emerged from the data codes were identified and organised into categories that could classify all information. A summative approach of the analysis was then performed which quantified the coded responses. ${ }^{11} \mathrm{~A}$ researcher $(\mathrm{xxx})$ led the identification of data codes, categorisation, and counting of recorded responses. These were reviewed in collaboration with another researcher (xxxx) to ensure consistency of interpretation with the description of responses. Verification of codes established was then investigated through a member checking exercise conducted using a focus group of allied health clinician volunteers from Monash Health. Data codes, categories, and their inter-relationships were presented to focus group participants by two researchers ( $x \mathrm{xx} \& \mathrm{xx}$.) along with exemplar quotes. Focus group participants discussed whether the naming of the codes and the inter-relationships reflected the data presented.

\section{RESULTS}

Ninety-five surveys were returned. The response rate was $13 \%$. Of the 95 respondents, eighty-six (90.5\%) were female. Their age distributions were $\mathrm{n}=41$ (43.2\%) between 30-39 years, 26 (27.4\%) between 40-49 years, 13 (13.7\%) between 50-59 years, 11 (11.6\%) between $20-29$ years, and four (4.2\%) aged over 60 years. Eighty-four reported their professional background. This included 27 physiotherapists (32.1\%), 23 occupational therapists $(27.4 \%)$, seven dieticians $(8.3 \%)$, six speech therapists $(7.1 \%)$, five social workers $(6 \%)$, five allied health assistants $(6 \%)$, four music therapists $(4.8 \%)$, three podiatrists $(3.6 \%)$, two neuropsychologists $(2.4 \%)$, one exercise physiologist $(1.2 \%)$ and one child life therapist $(1.2 \%)$. Out of the 94 respondents who reported their highest level of qualification, $53(56.4 \%)$ had a post-graduate qualification. Eighty-five reported their area of work. Twenty-nine (34.1\%) worked in acute inpatient services, $20(23.5 \%)$ in subacute inpatient services, $14(16.5 \%)$ in community health services, seven $(8.2 \%)$ in outpatient clinics, six $(7.1 \%)$ in specialist clinics, four $(4.7 \%)$ in mental health, four $(4.7 \%)$ in community rehabilitation and one $(1.2 \%)$ worked in domiciliary rehabilitation services.

\section{Description of the Highest Priority Research Area by Clinicians}

Overall, 23 of 95 respondents $(24.2 \%)$ provided a description of the highest priority research area that could be undertaken personally. Twenty-seven $(28.4 \%)$ provided a description of the highest priority research area that could be undertaken by their department or work areas, and $24(25.3 \%)$ provided a description of the highest priority research question that would need to be undertaken by the health service organisation.

\section{What is the highest priority research area at a personal level?}

Personal work evaluation. Many of the highest priority research projects that clinicians described and that could be undertaken individually were related to the evaluation of their personal work.

"Having worked with people with disabilities and their families and paid carers for 25 years, I feel I am well placed to work with a multidisciplinary team to investigate and evaluate interventions to improve patient experience and healthcare." (Neuropsychologist, participant no. 34)

\section{What is the highest priority research area at the departmental or work area level?}

Departmental or work area service evaluation. The highest priority research areas described by clinicians that could be undertaken by their department or work area were mostly related to the evaluation of the department-wide policies or procedures, or review of 
an entire profession's effectiveness in a given context. For example, one respondent identified need to review a physiotherapy department prioritisation tool, while another wanted to investigate the effectiveness of music therapy amongst stroke patients.

"Is music therapy effective in increasing arousal and orientation responses in patients presenting in a reduced state of consciousness in the acute stage of recovery following stroke?" (Music therapist, participant no.32)

\section{What is the highest priority research area across the health service organisational level?}

Models of care innovation. The highest priority research projects described by clinicians that would need to be undertaken across the health service sector were related to innovation of models of care. For example, inventing models of service delivery such as flexible care, interdisciplinary team and seamless care utilising same team across the continuum of care.

"Patients seen by the same allied health team across the continuum of care results in health and cost benefits to the patient and health network." (Physiotherapist, participant no.65)

\section{Categorisation of focus of highest priority research area}

The highest priority research identified at each level were categorised based on the nature of investigation that the provided response of research question seek to do. They were "finding problems," "understanding problems or developing solutions," "measuring problems or outcomes," "testing solutions," and "implementing evidence-based approaches in real life." Coded responses under each category for each level and for all levels combined are shown in Figure 1. The highest priority research investigation nominated by respondents across all levels combined was: "testing solutions" (46 coded responses); "understanding problem or developing solutions" (17 coded responses); "implementing evidence-based approaches in real life" (15 coded responses); "measuring problems or outcomes" (8 coded responses), and 'finding problems" (4 coded responses).

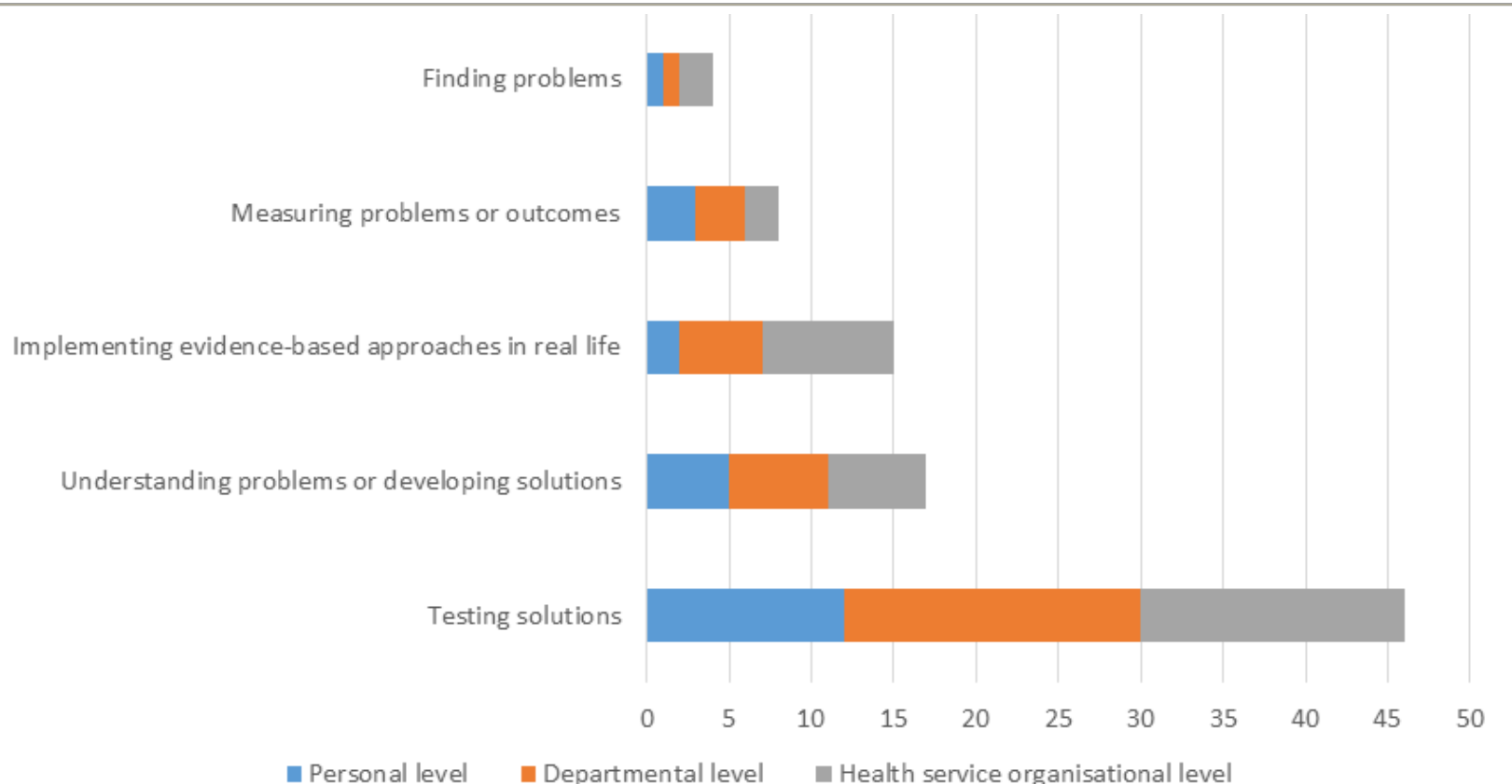

Figure 1. Areas of high priority research provided by clinicians across personal, departmental, and health service organisational levels (coded responses)

\section{Why were Different Research Areas Prioritised by Clinicians?}

Overall, 19 of 95 respondents (20\%) provided reasons for the highest priority research that could be undertaken personally, while $27(28.4 \%)$ respondents provided reasons for departmental or work area research. Twenty-five (26.3\%) respondents provided reasons for the highest priority research that would need to be undertaken across the organisation. Each respondent could provide more than one reason in each area. The most commonly reported reasons for each level and for all levels combined are illustrated (Figure 2). The underpinning reasons for the highest priority research projects across the three levels combined are described in more detail below. 


\section{Effectiveness of the intervention}

Respondents identified research areas as being a high priority primarily due to how effective they thought the intervention in question is or would be demonstrated to be. Many clinicians expressed the desire to evaluate their own professional impact within the broader service. Some felt the need to evaluate a hospital-based service provided to patients as it could impact on patients' care and improved their health outcomes.

"Often patients do not eat the food provided by the hospital due to their dislike for food which can exacerbate or worsen health conditions irrespective of medical treatment/intervention..." (Dietician, participant no. 18)

There were similar views expressed that research areas were a high priority because they would establish the effectiveness of departmental or allied health wide interventions or approaches. Some identified that certain roles of services may be underappreciated or at risk of losing support, yet be important for the recovery of patients. For example:

"This project would seek to demonstrate the direct and indirect role of hand therapy that plays a vital role in the plastics surgical service. This role is at times appreciated and underappreciated, yet remains vital for the patient journey. Demonstrating the hand therapist role may capture the attention of the powers that be in the service to increase funding for the vital role hand therapy plays and capture the brilliant work that hand therapy team completes." (Occupational therapist, participant no. 24)

\section{Cost-effectiveness of the intervention}

Another common justification for identifying a research area as a high priority was related to the anticipated cost-effectiveness of the solution being tested. Cost-effectiveness was conceptually related to the category of effectiveness but added the element of the relative cost to achieve the desired effect. Respondents often expressed this in terms of how their individual time was used, for example:

"To maximise efficiency of therapist to patient time." (Occupational therapist, participant no. 4)

Others discussed making improvements in service delivery that would save time across multiple staff members within a service, for example:

"Patients seen by the same allied health team across the continuum of care results in health and cost benefits to the patient and health network ... Improves patient care as well as reduces costs." (Physiotherapist, participant no. 65)

\section{Workforce roles}

A number of respondents identified particular professional groups or roles in their research areas, and justified the high priority ascribed on the need to demarcate the extent of these roles or professions within service delivery. This category was often related to both the effectiveness and cost-effectiveness categories, and to establishing career pathways for these professions or roles, for example:

"Do dedicated education roles in allied health increase student learning and education revenue compared to embedded supervisor model? ... Education should be an allied health career pathway. However, we largely have junior staff teaching students with potentially lesser education outcomes. Also dedicated education roles in community would be good to explore to increase capacity." (Physiotherapist, participant no. 91)

\section{Magnitude of the gap between evidence and practice}

Larger gaps between the evidence base and actual service delivery were given higher priority in several responses. For some respondents, this focused on increasing the amount of service relative to what was currently provided, but for others, it was about reducing or removing services that did not have a strong evidence base. This category was commonly related to both the categories of effectiveness and cost-effectiveness of the intervention, and at times, even workforce roles. For example:

"Disinvestment - what can we do without ... Ensures evidence based practice is prioritised. Important in the current economic environment...Supports evidence-based recruitment of staff." (Podiatrist, participant no. 72)

\section{Magnitude of health problem or unmet needs}

Respondents identified the magnitude of the health problem or the amount of unmet need increased the importance of a particular area. The magnitude of the problem or the need could be considered from the health service, caregiver or the patient's perspective. Respondents would refer to how common a particular problem was, or how much the problem impacted on individuals. For example: 
"Despite current evidence for clinical care we still have high rates of ulcer and amputation in this patient group. Nobody has done a review that looks at things from the patient perspective." (Podiatrist, participant 72)

\section{Other categories}

A range of other categories were identified, but were less commonly specified (Figure 2). Some related to the practical considerations related to conducting the proposed research, for example, "likelihood of attracting resources to conduct research", "involvement of multiple disciplines in conducting the research", and "resources required to conduct the research". Others related to organisational strategy and outcomes, for example, "alignment with organisational strategy", and "building reputation for allied health". There were some categories that were related to service, for example, "magnitude of resource involved in service provision", "enablement of measurement of impact", "access to service" and "assessing safety risk" of service. The remaining categories were more speculative and sought to prioritise project areas based on their "degree of innovation" and potential to "future proof the workforce". "Degree of innovation", "magnitude of resource involved in service provision" and "alignment with organisational strategy" were the categories that were exclusive to reasons provided for high priority research at the organisational level.

A common underlying motivator from a personal perspective of why respondents felt that particular research work should be done was driven by their passion and interest in that area.

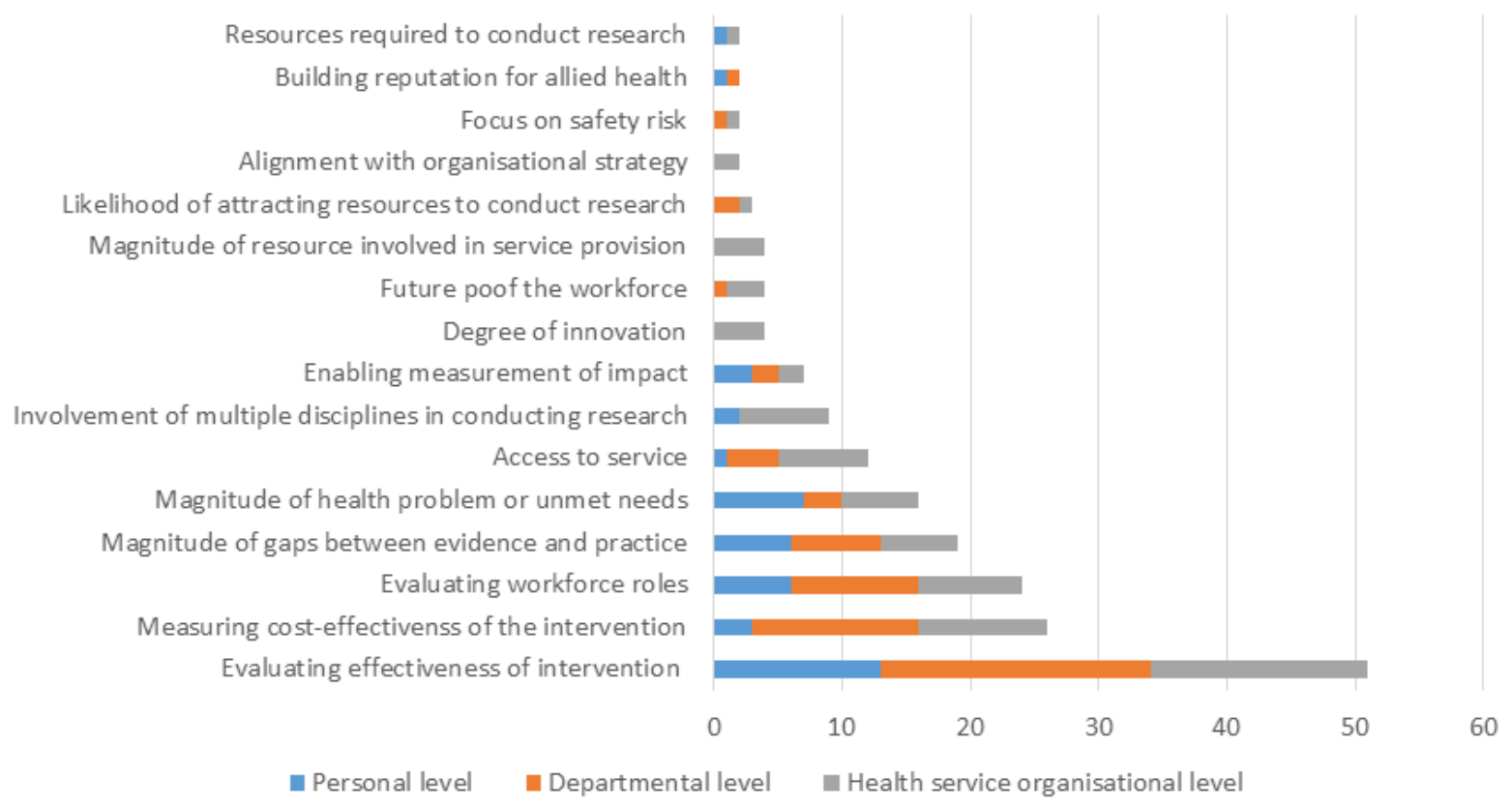

Figure 2. Reasons for high research priority area provided by allied health clinicians across personal, departmental, and health service organizational level (coded responses)

\section{Barriers and Enablers to Conducting High Priority Research}

There were 35 of 95 respondents (36.8\%) who provided responses for perceived barriers to the conduct of high priority research. Thirty-two $(33.7 \%)$ provided responses for perceived enablers. Each respondent could give more than one comment. There were eight categories of barriers identified. The largest category of barriers reported was "time shortage, workload and competing priorities" which consisted of 29 coded responses. There were seven categories of enablers identified. The largest category of enablers reported were "mentoring and accessing to university partners" and "organisational culture, leadership and priorities", both consisted of 10 coded responses. The key barriers and enablers were presented in Figure 3 and 4 . Examples of quotes within each of these categories of barriers and enablers are provided (Appendix 2). 
Bureaucracy/ap proval

Lack of local research support/relationship with academics

Lack of skill development/support

Lack of organisational support or culture

Lack of fin ancial support

Lack of departmental support

Time shortage/workload/competing priorities

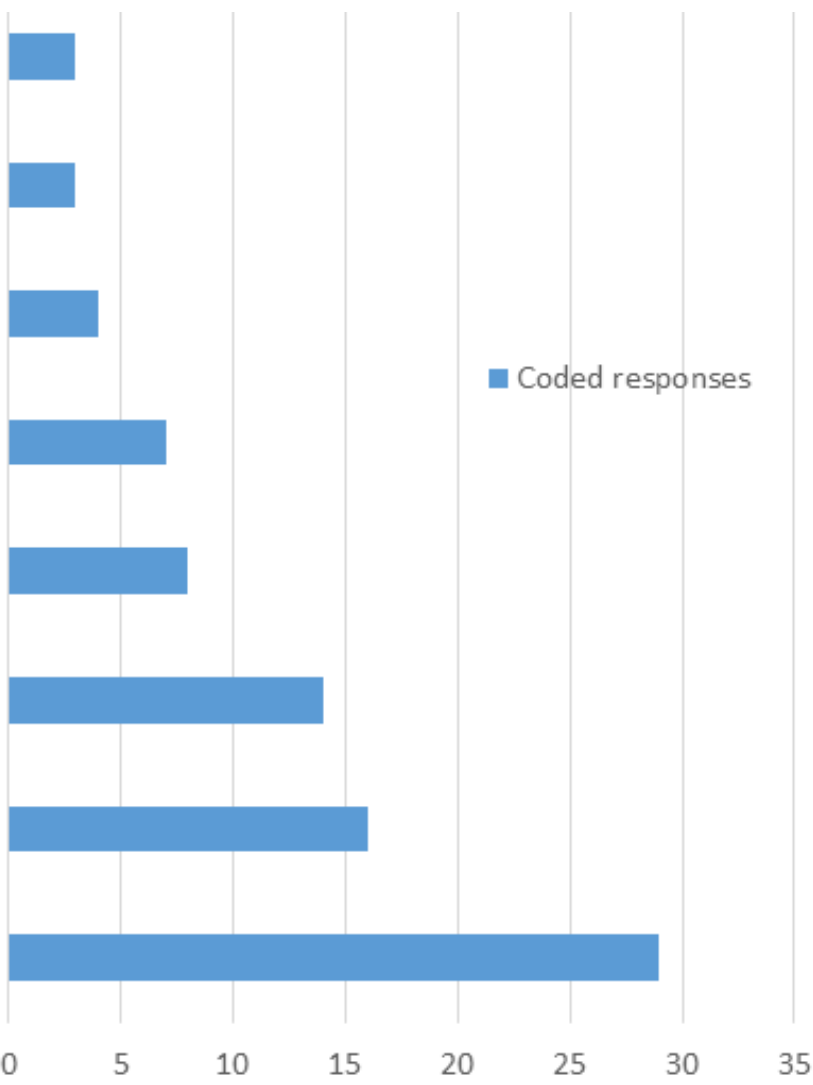

Figure 3. Barriers to conducting high priority research projects

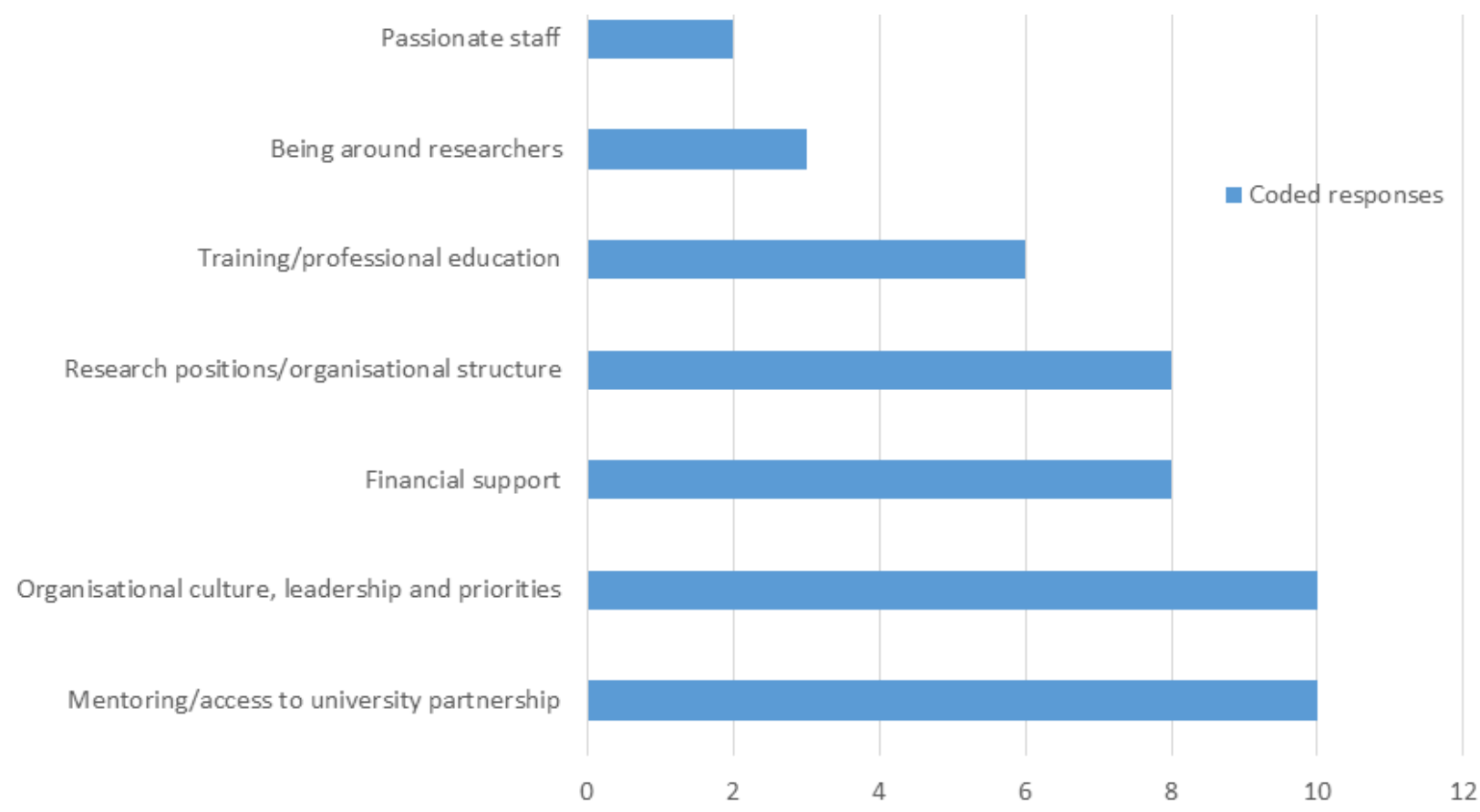

Figure 4. Enablers for conducting high priority research projects 


\section{DISCUSSION}

This research has created a picture of the types of research allied health staff are likely to try to pursue within a publicly funded health service and the reasons underpinning their areas of focus. Interestingly, our results showed clear preference for pursuing research focused upon "testing solutions" and that the potential effectiveness of interventions was a key factor driving research of this nature. Much less focus was given to research that could be considered to be essential in preparing for intervention studies, particularly research that seeks to understand problems or develop solutions, research identifying problems, and the measurement of problems or outcomes. This may be because clinicians feel they have a good understanding of the nature of the problems they deal with or how their potential solutions will work and can be implemented. However, there is a risk that intervention studies may be conducted in areas that are inadequately understood or using interventions that may not be the optimal solution for the problem at hand.

The most reported reasons provided for the highest priority research across all levels were the evaluation of effectiveness of interventions. This was closely connected to the perceived impact the respondents felt the research would have at each level across the organisational structure. For example, individuals expressed the need for evaluating their personal work practice, department focused on improving service provision through an evaluation of current practice. High priority research across the health service organisation was perceived to be capable of investigating the effectiveness of new and innovative models of care. All of these are consistent with what the clinicians identified to be the highest priority, and this was to test solutions to evaluate their effectiveness of work. Evaluation of cost-effectiveness of intervention and workforce roles were also highly reported reasons for high priority research across all three levels. These categories were inter-related, as an improvement in efficiency of clinical practice would allow greater ability to allocate staff to programs that may be understaffed. It can potentially inform workforce planning, create new roles, or change the scope of practice that is supported by research evidence. "Degree of innovation," "magnitude of resource involved in service provision," and "alignment with organisational strategy" were reasons provided for high priority research at the organisational level only. This may be because of the perception that the organisation has an overall executive and financial decision role for major changes, and a leadership role in steering allied health towards compliance with a strategic plan and policy of the organisation. An interesting point of contrast is the few comments being given on the "degree of innovation" as the reason for high priority research compared with innovation being one of the major funding criteria of nationally competitive funding schemes in Australia. ${ }^{12}$ It may be explained by the findings that the respondents viewed innovation of research was important at the organisational level but not for them personally. Innovation may be seen as a responsibility of the organisation. It can also be related to the solutions they wanted to test and that they were not considered to be innovative by the individual, rather, they were viewed as common sense solutions to everyday problems that they encountered.

Barriers identified by respondents for undertaking high priority research individually, across the department and organisation are predominantly extrinsic or external factors in their work environment. In particular "time shortage, heavy workload and prioritisation of clinical duties" is a key barrier identified. This is consistent with other studies. ${ }^{13,14}$ This key barrier is likely to be compounded by other barriers reported such as lack of support from an organisational, financial and research capacity viewpoints. ${ }^{15}$

In contrast to the barriers, the enablers being described were a combination of extrinsic and intrinsic factors. Although extrinsic factors are the major enablers, these were related to the availability of mentoring, having a research culture and leadership within the organisation, financial support and having a workforce structure that facilitates research. Intrinsic factors are those that are inherent to the individual and motivates them to undertake research. For example, a few respondents reported having clinicians that are passionate about research would enable the conduct of high priority research. This can be explained by the theories of motivation that suggest the conduct of research may satisfy the higher order needs for self-actualisation for some clinicians. ${ }^{16}$ This could explain clinicians often identified high priority research area to be those that were their own personal passion or interests.

Health care leaders may need to use a "top-down" approach to drive high priority research projects in publicly funded health care organisations that involves multiple clinicians and disciplines. However, organisations and their leaders can provide support to enhance the engagement of allied health clinicians to undertake the identified high priority research projects. Recommendations include providing research support; for example, giving support for time to be spent in research activities, allocating financial resources for research, building a culture for research through individual work area or department, increasing research capacity through training, and establishing research connections with academics.

There are limitations that need to be considered in this research. The survey was disseminated within Monash Health only. Therefore, the results may not be generalisable to other health service organisations including those that are privately funded in Australia or internationally. In addition, clinicians who have a higher interest in research may be more willing to participate in this kind of survey. This could potentially explain the low response rate that we experienced. 


\section{CONCLUSIONS}

Organisations may need to specifically identify, prioritise, and drive research that involves multiple clinicians, work areas, and/or disciplines using a top-down approach if they want this research to be undertaken. Considerations can be taken from our findings to guide the development of a framework or system to prioritise research projects in the publicly funded health care organisation. Further research will be beneficial to test the feasibility of the "top-down" approach to drive high priority research projects and the recommendations of support to promote this research in allied health disciplines in the publicly funded health care organisations.

\section{REFERENCES}

1. Cooke J, Nancarrow S, Hammersley V, Farndon L, Vernon W. The 'Designated Research Team' approach to building research capacity in primary care. Prim Health Care Res Dev. 2006;7(1):78-86. doi:10.1191/1463423606pc264oa

2. Ilott I, Bury T. Research Capacity: A challenge for the therapy professions. Physiotherapy. 2002;88(4):194-200. doi:10.1016/S0031-9406(05)60410-5

3. Ried K, Farmer EA, Weston KM. Setting directions for capacity building in primary health care: a survey of a research network. BMC Fam Pract. 2006;7(1):8. doi:10.1186/1471-2296-7-8 [PMID: 16466583]

4. Scotland N. Allied Health Professions: Research and development action Plan. In: Scotland N, ed. Edinburgh: Edinburgh: NHS Scotland; 2004.

5. Hodges P. Growth of physiotherapy research funding in Australia. Aust J Physiother. 2009;55. [PMID: 19681736]

6. Allied Health Professionals (Victorian Public Health Sector) Single Interest Entreprise Agreement 2016-2020. 2016; http://southwesthealthcare.com.au/assets/A/1230/9200d304d571266713077f6cc3e1599b/Attatchment\%20D\%20-\%20 Draft\%20EBA\%20Health\%20Professionals\%202016-2020.pdf. Accessed July 4, 2018.

7. Monash Health Fast Facts 2016-17 Website.http://www.monashhealth.org/page/fast_facts. Published March 8, 2018. Accessed July 23, 2018.

8. Holden L, Pager S, Golenko X, Ware RS. Validation of the research capacity and culture (RCC) tool: measuring RCC at individual, team and organisation levels. Aust J Prim Health. 2011;18(1):62-67. doi:10.1071/PY10081 [PMID: 22394664]

9. Da Costa Santos CM, de Mattos Pimenta CA, Nobre MRC. The PICO strategy for the research question construction and evidence search. Rev Lat Am Enfermagem. 2007;15(3):508. doi:10.1590/S0104-11692007000300023 [PMID: 17653438]

10. Anderson R. Thematic Content Analysis (TCA) Descriptive Presentation of Qualitative Data Using Microsoft Word. https://s3.amazonaws.com/academia.edu.documents/36098984/Thematic_Content_Analysis_manuscript.pdf?AWSAcc essKeyld=AKIAIWOWYYGZ2Y53UL3A\&Expires=1532325405\&Signature=giP3gDuQ9K9KUNn2hT5dy7IT4t4\%3D\&res ponse-content-disposition=inline\%3B\%20filename\%3DTHEMATIC_CONTENT_ANALYSIS_on_Microsoft_W.pdf. Accessed July 23, 2018.

11. Hsieh HF, Shannon SE. Three approaches to qualitative content analysis. Qual Health Res. 2005;15(9):1277-1288. doi:10.1177/1049732305276687 [PMID: 16204405]

12. NHMRC funding rules 2017. https://www.nhmrc.gov.au/book/nhmrc-funding-rules-2017. Accessed 4th July, 2018.

13. Cooke J, Owen J, Wilson A. Research and development at the health and social care interface in primary care: a scoping exercise in one National Health Service region. Health Soc Care Community. 2002;10(6):435-444. doi:10.1046/j.1365-2524.2002.00395.x [PMID: 12485130]

14. Pager S, Holden L, Golenko X. Motivators, enablers, and barriers to building allied health research capacity. J Multidiscip Healthc. 2012;5:53-59. doi.org/10.2147/JMDH.S27638 [PMID: 22396626]

15. Moore AP. Research, the Collaborative Approach: Clinicians and academics. Physiotherapy. 1997;83(5):229-234. doi:10.1016/S0031-9406(05)66212-8

16. Maslow AH. Motivation and personality. 3rd ed. / rev. by Robert Frager ... [et al.]. ed. New York: New York : Harper and Row; 1987. 
Appendix 1. A list of open-ended questions used in the survey instrument

1. Please describe the highest priority project that would need to be undertaken by allied health through multiple departments \&/or work areas across Monash Health. Remember to try and use the PICO format. Only one or two sentences are required. If you cannot think of one, enter "nil."

2. Please describe why you think this particular example of a project that would need to be undertaken by allied health through multiple departments \&/or work areas across Monash Health is the highest priority for you. You can provide as many reasons as you wish. If you could not think of an example for the question above, enter factors that you think should make a project a high priority project for allied health across Monash Health.

3. Please describe the highest priority project that could be undertaken by your department or work area. Remember to try and use the PICO format. Only one or two sentences are required. If you cannot think of one, enter "nil."

4. Please describe why you think this particular example of a project that could be undertaken by your department or work area is the highest priority for you. You can provide as many reasons as you wish. If you could not think of an example for the question above, enter factors that you think should make a project a high priority project for your department or work area.

5. Please describe the highest priority project that could be undertaken by yourself (individually) if you were going to lead a research project. Remember to try and use the PICO format. Only one or two sentences are required. If you cannot think of one, enter "nil."

6. Please describe why you think this particular example of a project that could be undertaken by yourself (individually) if you were going to lead a research project is the highest priority for you. You can provide as many reasons as you wish. If you could not think of an example for the question above, enter factors that you think would make a project a high priority project for yourself.

7. What are the barriers that you perceive stop high priority research from being conducted at Monash Health? Where relevant, specify if you feel a particular barrier is relevant to you leading your own research, to research that could be conducted through your department or work area, or to research that would need to be conducted through multiple departments \&/or work areas across Monash Health. You can provide as many barriers as you wish.

8. What are the enablers that you perceive facilitate high priority research to be conducted at Monash Health? Where relevant, specify if you feel a particular enabler is relevant to you leading your own research, to research that could be conducted through your department or work area, or to research that would need to be conducted through multiple departments \&/or work areas across Monash Health. You can provide as many enablers as you wish.

9. Please record any further comments or questions you have about conducting high priority research at Monash Health that you feel have not already been captured in your responses above. 
Appendix 2. Examples of quotes for the key barriers and enablers to the conduct of high priority research

\begin{tabular}{|c|c|c|c|}
\hline Barriers & Examples of quotes & Enablers & Examples of quotes \\
\hline $\begin{array}{l}\text { Time } \\
\text { shortage/workload/competing } \\
\text { priorities }\end{array}$ & $\begin{array}{l}\text { "Time, support, knowledge, } \\
\text { competing priorities...." } \\
\text { (Physiotherapist, participant } \\
\text { no.10) }\end{array}$ & $\begin{array}{l}\text { Mentoring/access to } \\
\text { university partnership }\end{array}$ & $\begin{array}{l}\text { "...access to experts, } \\
\text { strong links with } \\
\text { university partners" } \\
\text { (Physiotherapist, } \\
\text { participant no.40) }\end{array}$ \\
\hline Lack of departmental support & $\begin{array}{l}\text { "Little support from } \\
\text { department/profession...." } \\
\text { (Physiotherapist, participant } \\
\text { no.65) }\end{array}$ & $\begin{array}{l}\text { Organisational culture, } \\
\text { leadership and } \\
\text { priorities }\end{array}$ & $\begin{array}{l}\text { "Leadership, research } \\
\text { culture throughout ALL } \\
\text { areas of the } \\
\text { organisation..." } \\
\text { (Occupational therapist, } \\
\text { participant no.4) }\end{array}$ \\
\hline Lack of financial support & $\begin{array}{l}\text { "....money....." (Occupational } \\
\text { therapist, participant no.7) }\end{array}$ & Financial support & $\begin{array}{l}\text { "...access to small seed } \\
\text { grant..." } \\
\text { (Physiotherapist, } \\
\text { participant no. 16) }\end{array}$ \\
\hline $\begin{array}{l}\text { Lack of organisation support } \\
\text { or culture }\end{array}$ & $\begin{array}{l}\text { "There is not a culture or } \\
\text { expectation of research } \\
\text { amongst clinicians...... Allied } \\
\text { health clinical research } \\
\text { leadership is not visible to } \\
\text { clinicians...." (Occupational } \\
\text { therapist, participant no.4) }\end{array}$ & $\begin{array}{l}\text { Research positions } \\
\text { lorganisational } \\
\text { structure }\end{array}$ & $\begin{array}{l}\text { "the employment of } \\
\text { experienced } \\
\text { researchers....." } \\
\text { (Dietician, participant } \\
\text { no.2) }\end{array}$ \\
\hline Do not know what to do & $\begin{array}{l}\text { "...don't know where to start - } \\
\text { who to ask the question of?..." } \\
\text { (Physiotherapist, participant } \\
\text { no.65) }\end{array}$ & $\begin{array}{l}\text { Training/professional } \\
\text { education }\end{array}$ & $\begin{array}{l}\text { "Access to } \\
\text { knowledgeable } \\
\text { academics; support with } \\
\text { grant writing; } \\
\text { collaborating with } \\
\text { university staff..." } \\
\text { (Occupational therapist, } \\
\text { participant no.7) }\end{array}$ \\
\hline $\begin{array}{l}\text { Lack of skill } \\
\text { development/support }\end{array}$ & $\begin{array}{l}\text { "Skills: limited research skills } \\
\text { within my department; } \\
\text { knowledge: limited existing } \\
\text { research knowledge base at a } \\
\text { departmental level..." (Speech } \\
\text { therapist, participant no.27) }\end{array}$ & $\begin{array}{l}\text { Being around } \\
\text { researchers }\end{array}$ & $\begin{array}{l}\text { "Colleagues with an } \\
\text { interest in research such } \\
\text { as those completing } \\
\text { further study outside or } \\
\text { work..." (Physiotherapist, } \\
\text { participant no. 57) }\end{array}$ \\
\hline $\begin{array}{l}\text { Lack of local research } \\
\text { support/relationship with } \\
\text { academics }\end{array}$ & $\begin{array}{l}\text { "Few existing research } \\
\text { relationships with academic } \\
\text { institutions .....Departmental } \\
\text { support required to perceive } \\
\text { research as a part of an allied } \\
\text { health clinician's role..." (speech } \\
\text { therapist, participant no. 27) }\end{array}$ & Passionate staff & $\begin{array}{l}\text { "Motivated and } \\
\text { passionate staff about } \\
\text { research." (Music } \\
\text { therapist, participant } \\
\text { no.5) }\end{array}$ \\
\hline Bureaucracy/approval & $\begin{array}{l}\text { "Restrictions on structures to } \\
\text { support research in OT - hard to } \\
\text { get things off the ground; unsure } \\
\text { what the goals are; takes a long } \\
\text { time to get decisions made." } \\
\text { (Occupational therapist, } \\
\text { participant no. 7) }\end{array}$ & & \\
\hline
\end{tabular}

